\title{
Tailoring the TRIP effect of austenitic stainless steels with selective laser melting
}

\author{
Efthymios Polatidis ${ }^{1}$, Christos Sofras ${ }^{1}$, Capek Jan ${ }^{1}$, Ariyan Arabi-Hashemi ${ }^{2}$, Christian Leinenbach ${ }^{2}$, Markus Strobl ${ }^{1}$ \\ ${ }^{1}$ Paul Scherrer Institute, Villigen PSI, Switzerland; \\ ${ }^{2}$ Empa - Swiss Federal Laboratories for Materials Science and Technology, Dübendorf, Switzerland; \\ efthymios.polatidis@psi.ch
}

Laser Powder bed fusion (L-PBF) has attracted a lot of interest in recent years, not only for its profound advantage of producing metallic components of complex geometries but also for the possibility of manipulating microstructures and crystallographic textures. Additionally, recent observations on wrought austenitic steels have revealed the strong dependence of the transformation induced plasticity (TRIP) effect in metastable stainless steels on the crystallographic texture [1-3]. Taking the aforementioned observations into consideration, we can now process TRIP steels such as 304L by L-PBF, in order to produce differently textured specimens and manipulate the TRIP effect. In this contribution, in situ uniaxial tension and compression tests with neutron diffraction, are utilized for monitoring of the microstructural evolution during deformation. The present study highlights how different microstructures, produced by L-PBF, lead to different deformation behavior in austenitic stainless steels and paves the way for tailored microstructures in different types of steels and for studies under different loading conditions.

[1] E. Polatidis et al., "The interplay between deformation mechanisms in austenitic 304 steel during uniaxial and equibiaxial loading," 2019, doi: 10.1016/j.msea.2019.138222.

[2] E. Polatidis et al., "Suppressed martensitic transformation under biaxial loading in low stacking fault energy metastable austenitic steels," Scr. Mater., vol. 147, pp. 27-32, Apr. 2018, doi: 10.1016/j.scriptamat.2017.12.026.

[3] E. Polatidis, J. Čapek, A. Arabi-Hashemi, C. Leinenbach, and M. Strobl, "High ductility and transformation-induced-plasticity in metastable stainless steel processed by selective laser melting with low power," Scr. Mater., vol. 176, pp. 53-57, Feb. 2020, doi: 10.1016/j.scriptamat.2019.09.035.

Keywords: additive manufacturing, TRIP, austenitic steels, texture 